\title{
Un-motherly Mothers and Motherly Fathers: Gender Roles in Contemporary South Korean Reality Programs
}

Na Rim KIM

\section{Desperate "Celebrity" Housewives}

Featuring the everyday lives of "celebrity families" is a recent trend in South Korean reality programs. Such families consist of at least one celebrity parent and very young children. For celebrities this is a welcome trend, as it has given them the opportunity to present themselves as generally relatable despite their popularity. Due to the wide appeal of South Korean reality programs across diverse audiences-including young children, their parents, and even their grandparents-celebrity parents use these programs to reclaim the attention they lost after their marriage (Oh, 2014).

However, there is a catch: most featured celebrities are fathers; the few mothers who appear are often shown having a difficult time. For example: Mom Person (Park, 2015), the first program to exclusively feature celebrity mothers and their children, aired for only a month. This show focused on showing these celebrity mothers struggling to perform regular household chores. The struggle was almost painful to watch, so the show did not gather much interest and lasted for only four episodes. The three celebrity mothers on Mom Person wore little or no makeup, and looked tired, overwhelmed, and frustrated. Typical of its genre, Mom Person stressed its authenticity by often showing the mothers' tired faces-sometimes in extreme closeup-as they cook and clean for their young children. Some of these closeups are provided in Figure 1. 


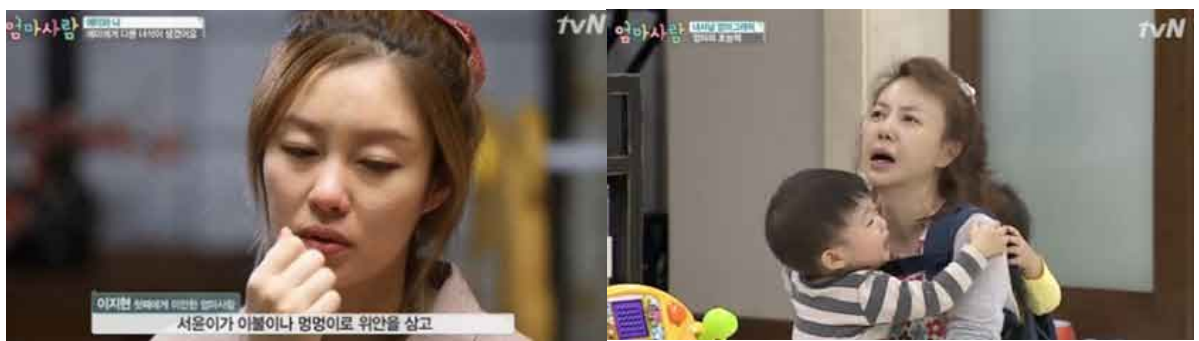

Figure 1. Left: The tired actress Ji-Hyun Lee (Kookje Paper, 2015); Right: Hye-Young Hwang looks exasperated with twins hitched on her front and back (Lee, 2015)

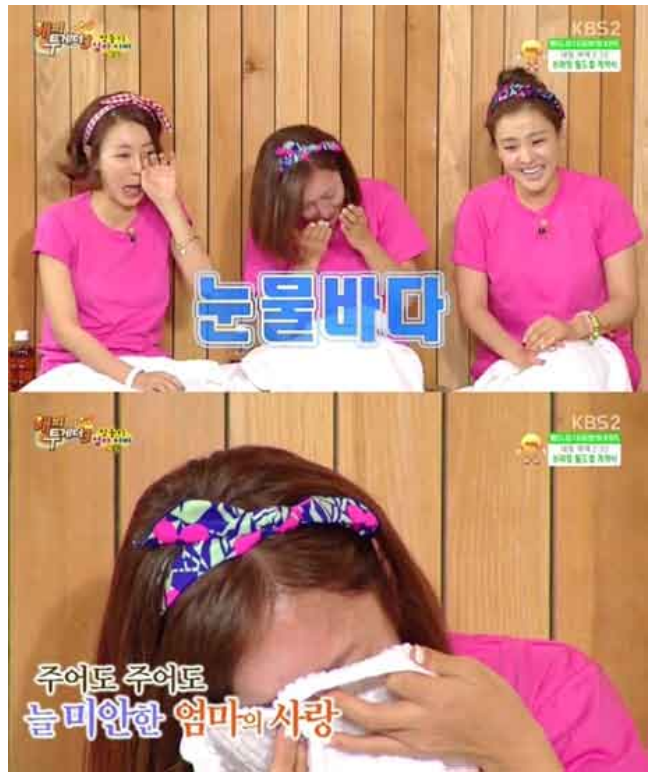

Figure 2. PSyu cries while talking about her son (Hwang, 2014).

Syu made two appearances on Happy Together: once in June and another in December. Syu was invited to discuss the hardships experienced by celebrity mothers. The director of the program emphasized Syu's grievances by captioning the segment "A sea of tears" (Figure 2, top), followed by the sentimental caption "Even though mothers give and give, a mother's love still always feels guilt towards their children" (Figure 2, bottom). The captions represent Syu as a guilty but loving mother. However: the public focused on Syu's guilt instead of how she loved her children.

Her appearances were followed by countless online news reports, which were posted almost directly after the shows were aired. Many articles depicted Syu as a mother who not only felt guilty, but as guilty of being an inadequate mother. For example: in an article regarding the episode aired in June, He-jin 
Hwang (2014) described how Syu "wailed" or "cried unrestrainedly" (para. 5) as she confessed to feeling guilty about not being a good enough mother for her twins. Readers expressed sympathy while criticizing how Syu "cried too much." When the public decided to withdraw its attention from Syu, only six articles reported her second appearance on the show in December, according to Naver, the major Korean search engine. As if conscious of the public's waning attention, these six articles chose to revert to the kind of yellow journalism that makes use of exaggerated and sensational content and titles. All six articles stress how Syu cried on the show and how her son took her food from her during a meal. Comments revealed how viewers criticized Syu for not being an understanding mother.

Regardless of how celebrities' personal distress always receives ample public attention, many viewers expressed discomfort towards Syu and her complaints about the difficulties of motherhood. Such responses fail to acknowledge how experiencing difficulties during child-rearing is a universal, natural experience for all parents.

\section{"Friendies":"Homely" Celebrity Fathers}

Compared to programs like Mom Person (Park, 2015) and the episodes of Happy Together (Park, 2007), programs featuring the struggles of celebrity fathers have steadily gained favorable reactions. One such popular show is The Return of Superman (Kim, 2013-), which continues its run in 2017 since it first aired in 2013. The "Superman" here refers to the celebrity fathers in the program who are left alone to watch their children while their wives leave for 48-hour vacation. The show documents four families and four fathers who discover how to become primary caregivers.

Another popular program featuring celebrity fathers acting as primary caregivers, Dad! where are we going? (Kwon, 2013-2015), launched in 2013. The father-centric program was very favorably received from its first

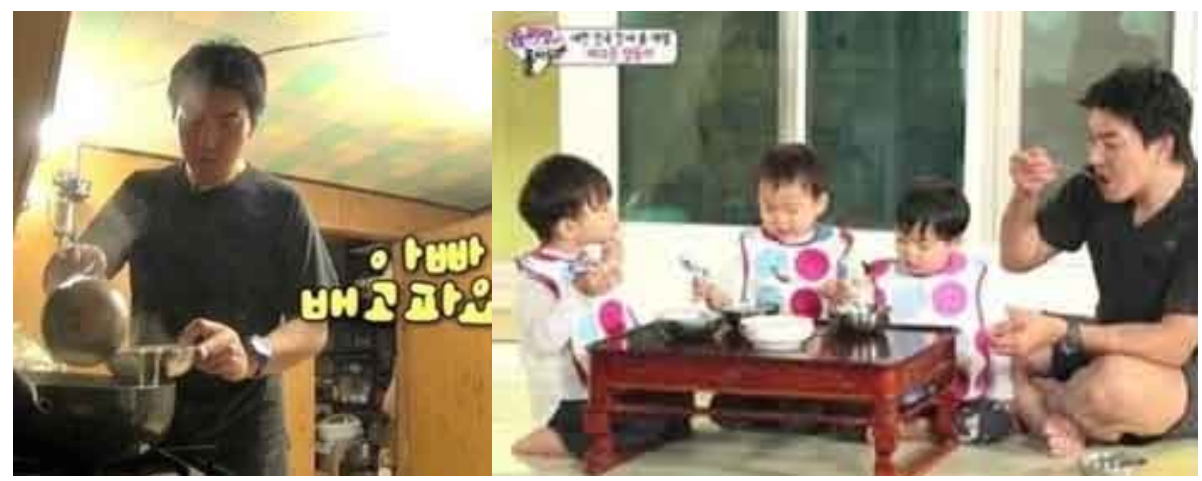

Figure 3. Actor II-Gook Song cooks for (left) then eats along with his triplets (right) (Kim, 2015) 
airing on September 19, 2013 until its final show on January 18, 2015 (Oh, 2014). ${ }^{1}$ Dad! where are we going? featured five Korean male celebrities and their children on trips to various rural locations. In these unfamiliar but interesting locations, the families engaged in activities the children had not experienced before, including: fishing, making fires, and camping out. Being the children of famous fathers, these children had not been able to spend much time with their respective fathers in the past. Hence the children greatly enjoyed the newfound time with their fathers. Although the program may have overemphasized sentimentality to gain more views, the father and child relationship noticeably improved.

Also noticeable was how the fathers became more adept at taking care of their children. As the program required camping outdoors, the fathers learned to cook. Due to a lack of experience, the food that they cooked for their children in the early episodes did not look very appetizing. However, as time progressed, their cooking skills also progressed.

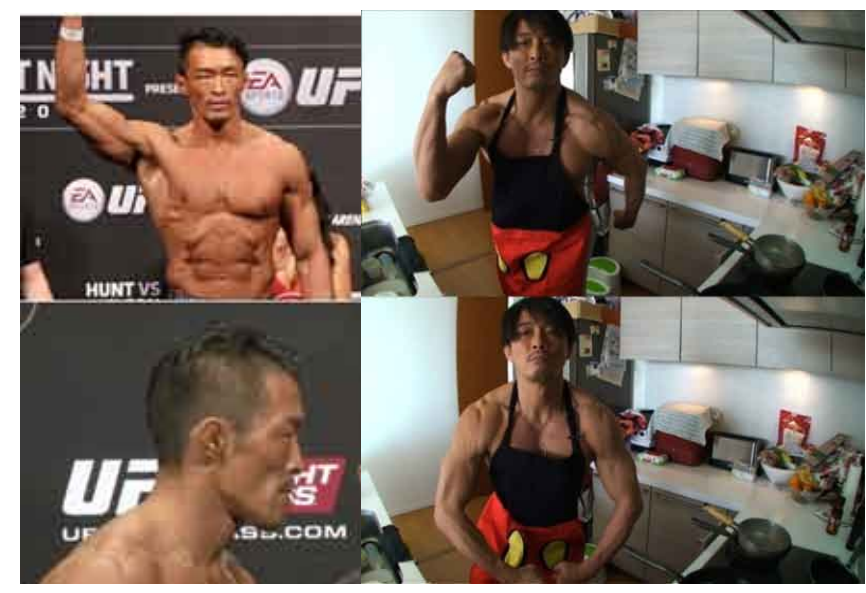

Figure 4. In the left picture Sung-Hoon $\mathrm{Chu}$ poses before a fight (Kim, 2014) and in the right he flashes his muscles in his kitchen while cooking for his daughter (Lee, 2013)

More recently, The Return of Superman (Kim, 2013) featured skilled fathers who were okay, even good, at cooking (Kim, 2015). Episodes for The Return of Supermen were shot in their actual homes (Figure 3), which made these men seem all the more domestic.

Noticeable among the cast was Sung-Hoon Chu, a champion mixed martial arts fighter. Viewers were surprised to see Sung-Hoon Chu act lovingly towards his child, cook, and clean adeptly in spite of his stereotypically masculine profession. Viewers complimented Chu for being both a loving and skilled father; Chu retained this image in spite of dropping out of the show. Images of Chu as a fierce fighter and as a gentle father have been juxtaposed in Figure 4 to give the reader an idea of how the Korean audience would have felt towards seeing Chu on The Return of Superman. 
Related to Chu's popularity is the wide usage within Korean mainstream culture of the terms "Ddal-babo," which literally means literally "a father who dotes on his daughter (Ddal) so much that he becomes stupid (Babo) when regarding matters concerning her," and the portmanteau "Friendy" from "Friend" and "Daddy" (Han, 2013). Chu openly doted on his daughter on television, which earned him both titles and also a sudden surge in popularity.

Hee-jeong Han claimed that the popular usage of these words was the result of attempts to change the Korean father image from the patriarchal father figure unable to communicate with family members to the loving father whose affections have been sadly misunderstood. Han (2013) specified that the image of the ideal father as depicted in Dad, where are we going? is "a father who does not repress or discipline their children but rather acts like a friend or a warm uncle" (p. 176).

Jang-guen Oh (2014) claimed that both Dad, where are we going? (Kwon, 2013) and The Return of Superman (Kim, 2013) stress the image of the loving father by always including interviews where celebrity fathers tell the audience that they have come to realize the joy of child-rearing and the importance of family. Oh (2014) also pointed out that non-celebrity fathers simply cannot reach the same conclusion because they rarely become primary caregivers for extended periods of time. Oh further criticized how the television programs represented a fathers's active role in childrearing as being uncommon. Oh challenged how the programs normalized a mother's return to primary caregiving and a father's return to their role as breadwinners.

Oh (2014) concluded that the efforts of these programs to replace the image of the authoritative father with a new, friendly image, did not lead to a deconstruction of patriarchal ideas. Rather, these shows ironically reinforced the traditional assumptions of the patriarchal system.

Seseoria Kim (2009) pointed out that the contemporary Korean concept of mothering expects mothers to "pour everything they have" (p. 33) into their children and find happiness and satisfaction in doing so. When celebrity fathers are depicted as finding tasks related to child care rewarding, mothers are encouraged even more to enjoy those tasks. For Korean mothers, childrearing is a challenge that stretches over an unspecified period of time, compared to the few celebrity fathers who appeared on reality programs to experience becoming a primary caregiver only twice a week. Now, celebrities are depicted as ordinary people with whom viewers are encouraged to identify. It is even more tempting to relate to celebrities when they are purposefully depicted as ordinary people by reality programs set in their respective residences. 


\section{Conclusion}

Celebrities were once mysterious objects for the public to fantasize about. Now, celebrities are depicted as ordinary people with whom viewers can identify. When the celebrities are depicted as ordinary people in reality programs set in their own residences, the audience relates to them. In their study of South Korean reality programs, Yeran Kim and Joo-Yeun Park (2006) found that such programs stress the accuracy of their representation of their audience's living situation (p. 37). The reality show directors disclosed that they saw themselves as "helping" the public by representing "real" situations that occur in "real" life, thereby allowing viewers to assess their lives through watching the shows. In other words, viewers would be presented with a standard against which they would be able to judge their degree of normalcy. Hence, the popular programs that feature celebrity families present a certain picture of what the "normal" South Korean family looks like.

South Korean reality programs that feature celebrity parents have recently tended to assign the traditionally female roles of cooking and taking care of children to male cast members. In doing so, these programs celebrate such roles and compliment the men who partake in them. The implication is that men watching the programs should follow suit. As such, if even the men are shown to be adept in these roles, women who have been in charge of household chores under a patriarchal system should have no problem. However, as seen with the example of Mom Person, women do have problems. When men can do "women's jobs" well, women who cannot do those jobs as well are an anomaly.

Citing how young women are generally better educated than older women, with better access to jobs, and with the option not to become housewives upon marriage, Jia Hong (2012) concluded that it is not fair to assume that more choices are available to Korean women of the current generation. As Hong saw it, young Korean women are still viewed primarily as potential housewives. He noted that contemporary Korean media most often represent women who are young and beautiful, and thus with the potential to become a submissive housewife. According to Korean media, young men who are economically well-to-do are the most sought after (Hong, 2012). Hong concluded that these standards reveal how women and men still occupy separate spheres. He adds that men occupy the social sphere as breadwinners; women are expected remain at home waiting to comfort their husbands with their soothing beauty.

Although South Korean reality television's depiction of men taking up traditionally feminine roles does show a different side of men, these representations do not sufficiently bridge the gender role divide. It is 
possible that programs depicting women enjoying professional roles could balance the trend. However, the challenge such programs would face is that South Korean reality television favors light, comic material. Hence, were such programs to be produced, the producers would need to take care and refrain from overly emphasizing the female cast as comic. 


\section{References}

Han, H. (2013). Audience reception of the representation of fatherhood in the reality TV program, addy! Where are we going? Social Science Research Review, 29(3), 161-186.

Hong, J. (2012). The way of love/to be loved suggested by TV and how 20's women in Korea interact with-focusing the <Mate>. Korean Journal of Broadcasting and Telecommunication Studies, 26(5), 307-342.

Hwang, H. (2014, June 13) Syu on Happy Together wails during shooting "I Feel Bad about My Twins" cries unrestrainedly. Retrieved from http://www.newsen.com/news_view. php?uid=201406122344020410.

Kim, H. (2013-). The Return of Superman [Television series]. Seoul: SBS.

Kim, H. (2014, December 26). Syu on Happy Together shed tears thinking of her son "He ate my food...I was hurt." Retrieved from http://www.fnnews.com/news/201412260032071588?t=p.

Kim, M. (2014, October 4) Superman returns, Sung-hoon Chu with split abs and fierce back muscle. Retrieved from http://news.topstarnews.net/detail.php?number=76367.

$\mathrm{Kim}, \mathrm{S}$. (2009).The meaning of mothering and women's work in the Korean family-focus on the children's education. Korean Feminist Philosophy, 12, 32-58.

Kim, Y. (2015, July 5). The Return of Superman, daddy's cooking show and kid's eating show draws attention. Retrieved from http://joynews.inews24.com/php/news_view.php?g_menu=700200\&g_ serial $=907078 \& \mathrm{rrf}=\mathrm{nv}$.

Kim,Y.\& Park,J.(2006). A study on theories and practices of reality programs. Korean Journal of Broadcasting and Telecommunication Studies, 20.3,7-48.

Lee, K. (2013, Dec. 27) Sung-hoon Chu transforms into Mickey Mouse, flashes muscles under an apron. Retrieved from http://news.joins.com/article/13499782.

Lee, M. \& Choi, Y. (2016). The exploratory study of relationship among motivations, gratification and viewing amount of Chinese remake"Dad! where are we going?" program. Journal of Communication Science, 16(1), 183-208.

Lee, W. (2015, April 1). Mom Person Ji-hyeon Lee shows tears as mother of two children. Retrieved from http://www.ytn.co.kr/_sn/1406_20150401223001928.

Mom Person Hye-young Hwang says "I'm happy, but raising kids takes guts." (2015, April 9). Kookje Paper. Retrieved from http://www.kookje.co.kr/news2011/asp/newsbody.asp?code=0540\&key=2015040 9.99002170314. (change in-text citation)

Oh, J. (2014). Korean family ideology in television entertainment programs:Dad! Where are we going? and The Return of Superman. Visual Culture, 25, 97-116.

Park, M. (2007-). [Television series]. Happy Together. Seoul: KBS.

\section{Note}

'The show has also been exported to China where the program was so popular that it aired for two seasons. After the first season ended, the reading rates of news articles reporting that a second season would follow the first season hit 19.8 billion views (Lee and Choi, 2016).

NA RIM KIM holds a Master's degree in English Language and Literature from Yonsei University in Seoul. She is currently directing her own research. Running through her work is an interest in how the individual's gender, race, and ethnicity affect that individual's self-understanding and relationships. (corresponding author: narim537@hanmail.net) 